\title{
FRACTIONAL DIFFERENTIATION AND LIPSCHITZ SPACES ON LOCAL FIELDS
}

BY

\author{
C. W. ONNEWEER
}

\begin{abstract}
In this paper we continue our study of differentiation on a local field $\mathbf{K}$. We define strong derivatives of fractional order $\alpha>0$ for functions in $L_{r}(K)$, $1<r<\infty$. After establishing a number of basic properties for such derivatives we prove that the spaces of Bessel potentials on $\mathbf{K}$ are equal to the spaces of strongly $L_{r}(\mathbf{K})$-differentiable functions of order $\alpha>0$ when $1<r<2$. We then focus our attention on the relationship between these spaces and the generalized Lipschitz spaces over K. Among others, we prove an inclusion theorem similar to a wellknown result of Taibleson for such spaces over $\mathbf{R}^{n}$.
\end{abstract}

1. Introduction. In [5] we presented a definition for differentiation of complexvalued functions on certain locally compact abelian groups and we gave a detailed comparison of this definition with earlier definitions due to Butzer and Wagner [3], Pál [6] and the present author [4]. In this paper we extend the definition given in [5] to differentiation of positive fractional order for functions defined on a local field $\mathbf{K}$.

In $\$ 2$ we introduce the necessary notation and we give the definition of fractional differentiation on $\mathbf{K}$. $\$ 3$ contains some of the standard properties for such derivatives that are needed later. In $\$ 4$ we prove that the spaces intermediate between $L_{r}(K), 1 \leqslant r \leqslant 2$, and $\mathscr{D}\left(D_{r}^{[\alpha]}\right)$, the space of functions in $L_{r}(K)$ that are strongly differentiable of order $\alpha>0$, are equal to the generalized Lipschitz spaces on $K$ as defined by Taibleson in [10]. In the final section we first identify the spaces $\mathscr{D}\left(D_{r}^{[\alpha]}\right)$ with the Bessel potential spaces on $\mathbf{K}$. This identification enables us to further elucidate the relationship between the generalized Lipschitz spaces and the spaces $\mathscr{D}\left(D_{r}^{[\alpha]}\right)$.

2. Notation. Let $\mathbf{N}, \mathbf{Z}, \mathbf{R}$ and $\mathbf{C}$ denote the natural numbers, the integers, the real numbers and the complex numbers, respectively, and let $\mathbf{K}$ denote a local field. Thus, $\mathbf{K}$ is a locally compact, totally disconnected, nondiscrete field. Let $\boldsymbol{P}_{\mathbf{0}}$ be the ring of integers of $\mathbf{K}$ and let $P$ be the (unique) maximal ideal in $P_{0}$. Furthermore, let $\mathfrak{p}$ be an element of $P$ that generates $P$. Then $P_{0} / P \simeq \mathrm{GF}(q)$, where $q$ is the power of a prime number $p$. Also, a nonarchimedean norm can be defined on $K$ which we denote by $\|\cdot\|$. For each $k \in \mathbf{Z}$ we set

$$
P_{k}=\left\{x \in \mathbf{K} ;\|x\| \leqslant q^{-k}\right\} \text {. }
$$

Received by the editors March 5, 1979; presented in part to the Society, June 16, 1979 under the title Bessel potentials and Lipschitz spaces on local fields.

AMS (MOS) subject classifications (1970). Primary 43A70, 26A24; Secondary 26A33, 28 A15.

Key words and phrases. Local fields, fractional derivatives, Bessel potentials, generalized Lipschitz spaces. 
Then $P_{k}=\mathfrak{p}^{k} P_{0}$, each $P_{k}$ is a subgroup of $\mathbf{K}^{+}, \mathbf{K}^{*}$ considered as an additive group, and these subgroups are a complete system of neighborhoods of $0 \in \mathbf{K}$.

Let $m$ or $d x$ denote Haar measure on $\mathbf{K}$, normalized so that $m\left(P_{0}\right)=1$. Then $m\left(P_{k}\right)=q^{-k}$ for each $k \in \mathbf{Z}$.

The dual group $\hat{\mathbf{K}}$ of $\mathbf{K}^{+}$can be described as follows. Choose a character $\chi \in \hat{\mathbf{K}}$ such that $\chi(x)=1$ for all $x \in P_{0}$ and $\chi(x) \neq 1$ for some $x \in P_{-1}$. Then $\mathbf{K}=\left\{\chi_{y}\right.$; $y \in \mathbf{K}\}$, where $\chi_{y}$ is defined by $\chi_{y}(x)=\chi(y x)$ for all $x \in \mathbf{K}$. For $f \in L_{r}(\mathbf{K})$, $1<r<2$, the Fourier transform $\hat{f}$ is defined the usual way, see [11, Chapter II, $\S \S 1,2]$; we use the notation $\hat{f}(y)$ for $\hat{f}\left(x_{y}\right)$. For each $k \in \mathbf{Z}$ the function $\Delta_{k}$ on $\mathbf{K}$ is defined by

$$
\Delta_{k}(x)= \begin{cases}q^{k} & \text { if } x \in P_{k} \\ 0 & \text { if } x \notin P_{k}\end{cases}
$$

Then, see [11, p. 23], we have

$$
\left(\Delta_{k}\right)^{\wedge}(y)= \begin{cases}1 & \text { if } y \in P_{-k}, \\ 0 & \text { if } y \notin P_{-k}\end{cases}
$$

For additional information and results on harmonic analysis on $\mathbf{K}$ we refer to the papers [9] and [10] and the lecture notes [11] by Taibleson. Note, however, that Taibleson uses the notation $R(x,-k)$ for the functions $\Delta_{k}(x)$ defined in (1).

We now present our definition of differentiation of fractional order on $\mathbf{K}$.

Definition 1. For $f \in L_{1, \text { loc }}(\mathbf{K}), \alpha>0, m \in \mathbf{N}$ and $x \in \mathbf{K}$ let

$$
E_{m, \alpha} f(x)=\sum_{l=-\infty}^{m-1}\left(q^{(l+1) \alpha}-q^{l \alpha}\right)\left(f-\Delta_{l} * f\right)(x) .
$$

(a) If $\lim _{m \rightarrow \infty} E_{m, a} f(x)$ exists, the limit is called the pointwise derivative of order $\alpha$ of $f$ at $x$, denoted by $f^{[\alpha]}(x)$.

(b) If $f \in L_{r}(\mathbf{K}), 1<r<\infty$, or $f \in C(\mathbf{K})$ and if $\lim _{m \rightarrow \infty} E_{m, a} f$ exists in the strong sense, the limit is called the strong derivative of order $\alpha$ of $f$, denoted by $D_{r}^{[\alpha]} f$ if $f \in L_{r}(\mathbf{K})$ and by $D_{C}^{[\alpha]} f$ if $f \in C(\mathbf{K})$.

We set $\mathscr{D}\left(D_{r}^{[\alpha]}\right)=\left\{f \in L_{r}(\mathbf{K}) ; D_{r}^{[\alpha]} f\right.$ exists $\}$.

RemarK. For $\alpha=1$ this definition agrees with Definition 3 of [5] applied to $K$. It is clear from [5] that, after some minor modifications, the present definition can also be applied to groups other than $\mathbf{K}^{+}$. However, since presently we are mainly interested in applications on local fields we have restricted ourselves from the outset to this case.

3. Basic properties of fractional derivatives. In this section we present some of the standard properties of derivatives of fractional order on local fields. The first result indicates that such derivatives are similar to the Riesz derivatives of fractional order on $\mathbf{R}$ or $\mathbf{T}$, the circle group. Additional evidence for this fact is contained in Theorem 3. 
THEOREM 1. (a) For each $\alpha>0$ and $x, y \in \mathbf{K}, \chi_{y}^{[\alpha]}(x)$ exists and $\chi_{y}^{[\alpha]}(x)=$ $\|y\|^{\alpha} \chi_{y}(x)$.

(b) For each $\alpha>0, k \in \mathbf{Z}$ and $x \in \mathbf{K}, \Delta_{k}^{[\alpha]}(x), D_{r}^{[\alpha]} \Delta_{k}, 1<r<\infty$, and $D_{C}^{[\alpha]} \Delta_{k}$ exist and

$$
\Delta_{k}^{[\alpha]}(x)=\sum_{l=-\infty}^{k} q^{l \alpha}\left(\Delta_{l}-\Delta_{l-1}\right)(x) .
$$

$A$ similar equality holds for the strong derivatives of $\Delta_{k}$.

(c) For each $f \in L_{r}(\mathbf{K}), 1 \leqslant r<\infty$, each $\alpha>0$ and $k \in \mathbf{Z}$ we have $\Delta_{k} * f \in$ $\mathscr{D}\left(D_{r}^{[\alpha]}\right)$ and

$$
D_{r}^{[\alpha]}\left(\Delta_{k} * f\right)=D_{r}^{[\alpha]}\left(\Delta_{k}\right) * f=\sum_{l=-\infty}^{k} q^{l \alpha}\left(\Delta_{l}-\Delta_{l-1}\right) * f
$$

Proof. Part (a) can be proved by a straightforward computation of the $E_{m, \alpha} \chi_{y}(x)$, compare [5, Theorem 3(a)]. To prove (b), fix $k \in \mathbf{Z}$. It follows from (1) that for each $l \in \mathbf{Z}$ and $x \in \mathbf{K}$ we have $\Delta_{l} * \Delta_{k}(x)=\Delta_{l \wedge k}(x)$, where $l \wedge k=$ $\min \{k, l\}$. Consequently, for $m>k$ we have

$$
\begin{aligned}
E_{m, \alpha} \Delta_{k}(x) & =\sum_{l=-\infty}^{k-1}\left(q^{(l+1) \alpha}-q^{l \alpha}\right)\left(\Delta_{k}-\Delta_{l}\right)(x) \\
& =\sum_{l=-\infty}^{k} q^{l \alpha}\left(\Delta_{l}-\Delta_{l-1}\right)(x) .
\end{aligned}
$$

Since the series defining $E_{m, \alpha} \Delta_{k}(x)$ converges and is independent of $m$ we have (b). A proof of (c) can be given using the same argument as was given in (b).

Let $\mathcal{S}$ denote the set of test functions on $K$ as defined in [9] or [11], thus $f \in \mathcal{S}$ if and only if $f$ is a finite linear combination of translates of the functions $\Delta_{k}(k \in Z)$.

Corollary 1. Every $f \in \mathcal{S}$ is differentiable of order $\alpha$ for all $\alpha>0$, both pointwise and in the strong sense.

For the remainder of this paper we mainly consider strong differentiability for functions in $L_{r}(\mathbf{K}), 1 \leqslant r \leqslant 2$. The reason for this restriction on $r$ is that presently we do not want to consider functions whose Fourier transform may only be defined in the distributional sense. We hope to deal with the latter case elsewhere. For later reference we state some theorems which generalize results obtained previously in [5] for the case $\alpha=1$ and $r=1$. When no proofs are given we refer to the proofs of the corresponding results in [5].

Theorem 2. For $f \in L_{r}(\mathbf{K}), 1 \leqslant r \leqslant 2, \alpha>0, m \in \mathbf{N}$ and a.e. $y \in \mathbf{K}$ we have

$$
\left(E_{m, \alpha} f\right)^{\wedge}(y)= \begin{cases}\|y\|^{\alpha} \hat{f}(y) & \text { if } y \in P_{-m}, \\ q^{m \alpha} \hat{f}(y) & \text { if } y \notin P_{-m} .\end{cases}
$$

The next theorem gives a characterization for $\mathscr{D}\left(D_{r}^{[\alpha]}\right)$ when $\alpha>0$ and $1<r<$ 2. First, however, we state a lemma that is needed in the proof of Theorem 3. This 
result is a simple extension of Lemma 2 in [4] and can be proved by the same method that was used there.

LEMMA 1. For each $k \in \mathbf{Z}, \alpha>0$ and $x \neq 0$, define $V_{k, \alpha}(x)$ by

$$
V_{k, \alpha}(x)=\sum_{l=-k+1}^{\infty} q^{-l \alpha}\left(\Delta_{l}-\Delta_{l-1}\right)(x) .
$$

Then $V_{k, \alpha} \in L_{1}(\mathbf{K})$ and $\left\|V_{k, \alpha}\right\|_{1}=O\left(q^{k \alpha}\right)$. Moreover,

$$
\left(V_{k, \alpha}\right)^{\wedge}(y)= \begin{cases}0 & \text { if } y \in P_{k}, \\ \|y\|^{-\alpha} & \text { if } y \notin P_{k} .\end{cases}
$$

We use the following notation:

$$
\begin{aligned}
& W\left(L_{r},\|y\|^{\alpha}\right)=\left\{f \in L_{r}(\mathbf{K}) ; \text { there is a } g \in L_{r}(\mathbf{K})\right. \text { such that } \\
& \left.\qquad \hat{g}(y)=\|y\|^{\alpha} \hat{f}(y) \text { a.e. on } \mathbf{K}\right\} .
\end{aligned}
$$

Theorem 3. For $\alpha>0$ and $1<r \leqslant 2$ we have $\mathscr{D}\left(D_{r}^{[\alpha]}\right)=W\left(L_{r},\|y\|^{\alpha}\right)$.

Proof. For $r=1$ and $\alpha>0$ the proof is essentially the same as that given in [5] for $r=1$ and $\alpha=1$. Thus, we shall restrict ourselves to $1<r<2$. First, take an $f \in \mathscr{D}\left(D_{r}^{[\alpha]}\right)$. Then there exists a $g \in L_{r}(\mathbf{K})$ so that

$$
\lim _{m \rightarrow \infty}\left\|E_{m, \infty} f-g\right\|_{r}=0 \text {. }
$$

Also, Theorem 2 implies that for a.e. $y \in \mathbf{K}$ we have

$$
\lim _{m \rightarrow \infty}\left(E_{m, \alpha} f\right)^{\wedge}(y)=\|y\|^{\alpha} \hat{f}(y) \text {. }
$$

Therefore, it follows from Fatou's lemma and the Hausdorff-Young inequality that, with $(r)^{-1}+\left(r^{\prime}\right)^{-1}=1$,

$$
\begin{aligned}
\|\| \cdot\left\|^{\alpha} \hat{f}(\cdot)-\hat{g}(\cdot)\right\|_{r^{\prime}} & \leqslant \liminf _{m \rightarrow \infty}\left\|\left(E_{m, \alpha} f\right)^{\wedge}-\hat{g}\right\|_{r^{\prime}} \\
& \leqslant \liminf _{m \rightarrow \infty}\left\|E_{m, \alpha} f-g\right\|_{r}=0 .
\end{aligned}
$$

Thus $\hat{g}(y)=\|y\|^{\alpha} \hat{f}(y)$ a.e. on $\mathbf{K}$, that is, $f \in W\left(L_{r},\|y\|^{\alpha}\right)$. Conversely, take an $f \in W\left(L_{r},\|y\|^{\alpha}\right)$ and assume $g \in L_{r}(\mathbf{K})$ satisfies $\hat{g}(y)=\|y\|^{\alpha} \hat{f}(y)$ a.e. on $\mathbf{K}$. Then a comparison of the Fourier transforms and the uniqueness theorem shows that

$$
E_{m, \alpha} f=\Delta_{m} * g+q^{m a} V_{-m, \alpha} *\left(g-\Delta_{m} * g\right)
$$

Therefore,

$$
\left\|E_{m, \alpha} f-g\right\|_{r} \leqslant\left\|\Delta_{m} * g-g\right\|_{r}+q^{m \alpha}\left\|V_{-m, \alpha}\right\|_{1}\left\|\Delta_{m} * g-g\right\|_{r} .
$$

Thus, using the estimate for $\left\|V_{-m, \alpha}\right\|_{1}$ mentioned in Lemma 1 and the fact that $\lim _{m \rightarrow \infty}\left\|\Delta_{m} * g-g\right\|_{r}=0$, we see that $\lim _{m \rightarrow \infty}\left\|E_{m, \alpha} f-g\right\|_{r}=0$, that is, $f \in \mathscr{D}\left(D_{r}^{[\alpha]}\right)$.

Once we have Theorem 3 it is easy to prove

Corollary 2. For $\alpha>0$ and $1<r<2$ the operator $D_{r}^{[\alpha]}$ is a closed linear operator on $\mathscr{D}\left(D_{r}^{[\alpha]}\right)$. 
Consequently, we can define a norm on $\mathscr{D}\left(D_{r}^{[\alpha]}\right)$ which makes this space into a Banach space; we denote this norm by $\|\cdot\|_{D(r, \alpha)}$ :

$$
\|f\|_{D(r, \alpha)}=\|f\|_{r}+\left\|D_{r}^{[\alpha]} f\right\|_{r} .
$$

Before stating the last theorem of this section we mention some results proved in [9] or [11]. For each $\alpha \in C$ there exists a distribution $G_{\alpha} \in \mathcal{S}^{\prime}$ whose Fourier transform, in the distributional sense, is given by

$$
\left(G_{\alpha}\right)^{\wedge}(y)= \begin{cases}1 & \text { if } y \in P_{0}, \\ \|y\|^{-\alpha} & \text { if } y \notin P_{0} .\end{cases}
$$

Moreover, if $\operatorname{Re} \alpha>0$ then, actually, $G_{\alpha} \in L_{1}(\mathbf{K})$ and $\left\|G_{\alpha}\right\|_{1}=1$. Also, in [9, Theorem 5] or [11, p. 142] Taibleson proved that if $\operatorname{Re} \alpha>0$ then $\|y\|^{\alpha}=$ $\left(\mu_{\alpha}\right)^{\wedge}(y)\left(G_{-\alpha}\right)^{\wedge}(y)$. Here $\mu_{\alpha}$ is the Borel measure on $\mathbf{K}$ defined by

$$
\mu_{\alpha}=\delta_{0}-\Delta_{0}+D{ }^{\alpha]} \Delta_{0}
$$

where $\delta_{0}$ is the Dirac $\delta$-measure concentrated at $0 \in \mathbf{K}$. Observe that it follows from (2) and Theorem 3 that

$$
\left(\mu_{\alpha}\right)^{\wedge}(y)= \begin{cases}\|y\|^{\alpha} & \text { if } y \in P_{0}, \\ 1 & \text { if } y \notin P_{0} .\end{cases}
$$

TheOREM 4. If $1 \leqslant r \leqslant 2$ and $0<\beta<\alpha$ then $\mathscr{D}\left(D_{r}^{[\alpha]}\right) \subset \mathscr{D}\left(D_{r}^{[\beta]}\right)$; the inclusion is continuous.

Proof. Let $f \in \mathscr{D}\left(D_{r}^{[\alpha]}\right)$ and let $g=D_{r}^{[\alpha]} f$. Then

$$
\begin{aligned}
\|y\|^{\beta} \hat{f}(y) & =\left(\mu_{\beta}\right)^{\wedge}(y)\left(G_{-\beta}\right)^{\wedge}(y) \hat{f}(y) \\
& =\left(\mu_{\beta}\right)^{\wedge}(y)\left(G_{\alpha-\beta}\right)^{\wedge}(y)\left(G_{-\alpha}\right)^{\wedge}(y) \hat{f}(y) \\
& =\left(\mu_{\beta}\right)^{\wedge}(y)\left(G_{\alpha-\beta}\right)^{\wedge}(y)\left(\|y\|^{\alpha}+\left(\Delta_{0}-\left.D\right|^{\alpha]} \Delta_{0}\right)^{\wedge}(y)\right) \hat{f}(y) \\
& =\left(\mu_{\beta}\right)^{\wedge}(y)\left(G_{\alpha-\beta}\right)^{\wedge}(y)\left(\hat{g}(y)+\left(\Delta_{0}-\left.D\right|^{\alpha]} \Delta_{0}\right)^{\wedge}(y) \hat{f}(y)\right) .
\end{aligned}
$$

Thus, since $\mu_{\beta}$ is a finite Borel measure, $G_{\alpha-\beta} \in L_{1}(\mathbf{K})$ and $g+\left(\Delta_{0}-D_{1}^{[\alpha]} \Delta_{0}\right) * f \in$ $L_{r}(\mathbf{K})$, we see that $\|y\|^{\beta} \hat{f}(y)$ is the Fourier transform of a function in $L_{r}(K)$, that is, $f \in W\left(L_{r},\|y\|^{\beta}\right)$. According to Theorem 3 this is equivalent to $f \in \mathscr{D}\left(D_{r}^{[\beta]}\right)$. Also, it follows from

$$
D_{r}^{[\beta]} f=\mu_{\beta} * G_{\alpha-\beta} *\left(D_{r}^{[\alpha]} f+\left(\Delta_{0}-D_{l}^{[\alpha]} \Delta_{0}\right) * f\right)
$$

that

$$
\begin{aligned}
\|f\|_{D(r, \beta)} & =\|f\|_{r}+\left\|D_{r}^{[\beta]} f\right\|_{r} \\
& \leqslant\|f\|_{r}+\left\|\mu_{\beta}\right\|_{M}\left\|G_{\alpha-\beta}\right\|_{1}\left(\left\|D_{r}^{[\alpha]} f\right\|_{r}+\left(\left\|\Delta_{0}\right\|_{1}+\left\|D^{[\alpha]} \Delta_{0}\right\|_{1}\right)\|f\|_{r}\right) \\
& \leqslant\|f\|_{r}+C_{\beta}\left(\left\|D_{r}^{[\alpha]} f\right\|_{r}+C_{\alpha}\|f\|_{r}\right) \\
& =C_{\alpha, \beta}\left(\|f\|_{r}+\left\|D_{r}^{[\alpha]} f\right\|_{r}\right)=C_{\alpha, \beta}\|f\|_{D(r, \alpha)} .
\end{aligned}
$$

This proves the continuity of the inclusion relation and completes the proof of Theorem 4. 
4. Intermediate spaces for $\mathscr{D}\left(D_{r}^{[\alpha]}\right)$ and $L_{r}(K)$. In this section we identify the spaces that are obtained as intermediate spaces between $\mathscr{D}\left(D_{r}^{[\alpha]}\right)$ and $L_{r}(\mathbf{K})$ by means of Peetre's so-called $K$-method. For the definition and basic properties of this method, see [1] or [2]. We shall prove that these intermediate spaces are precisely the generalized Lipschitz spaces on $K$ as defined by Taibleson in [10], or $[11$, p. 180]. We repeat this definition here.

Definition 2. Let $1 \leqslant r, s \leqslant \infty$ and $\alpha>0 . \Lambda(\alpha, r, s)$ is the space of all $f \in$ $L_{r}(\mathbf{K})$ for which any of the following three equivalent norms is finite:

(a) $\|f\|_{r}+\left(\int_{K}\|\| y\left\|^{-\alpha}(f(\cdot+y)-f(\cdot))\right\|_{r}^{s}\|y\|^{-1} d y\right)^{1 / s}$,

(b) $\|f\|_{r}+\left(\sum_{k=-\infty}^{\infty}\left\|q^{k \alpha}\left(f-\Delta_{k} * f\right)\right\|_{r}^{s}\right)^{1 / s}$,

(c) $\|f\|_{r}+\left(\sum_{k=-\infty}^{\infty}\left\|q^{k \alpha}\left(\Delta_{k+1}-\Delta_{k}\right) * f\right\|_{r}^{s}\right)^{1 / s}$,

with the usual change if $s=\infty$. We shall denote, with some abuse of notation, any of these three expressions by $\|f\|_{\Lambda(\alpha, r, s)}$.

TheOREM 5. For $1 \leqslant r \leqslant 2,1 \leqslant s \leqslant \infty$ and $0<\beta<\alpha$ we have

$$
\left(L_{r}(\mathbf{K}), \mathscr{D}\left(D_{r}^{[\alpha]}\right)\right)_{\beta / \alpha, s ; K}=\Lambda(\beta, r, s)
$$

This theorem is an immediate consequence of the following result due to Butzer and Scherer [2, Theorem 4.1]. Note that here and elsewhere $C$ will denote a constant, not necessarily having the same value throughout an argument.

TheOREM (BUTZER AND SCHERer). Let $X$ be $a$ Banach space and $Y$ a Banach subspace of $X$. Let $\left\{U_{k}\right\}_{1}^{\infty}$ be a sequence of bounded commutative linear transformations of $X$ such that there exists constants $C>0$ and $\alpha>0$ for which

(i) $\lim _{k \rightarrow \infty} U_{k} f=f$ for $f \in X$,

(ii) $\left\|U_{k} f-f\right\|_{X} \leqslant C k^{-\alpha}\|f\|_{Y}$ for $f \in Y$,

(iii) $U_{k} f \in Y$ and $\left\|U_{k} f\right\|_{Y} \leqslant C k^{\alpha}\|f\|_{X}$ for $f \in X$.

If $0<\beta<\alpha$ and $1<s \leqslant \infty$ then the following conditions are equivalent:

(a) $f \in(X, Y)_{\beta / \alpha, s ; K}$,

(b) $\|f\|_{X}+\left(\sum_{k=1}^{\infty}\left(k^{\beta}\left\|U_{k} f-f\right\|_{X}\right)^{s} k^{-1}\right)^{1 / s}<\infty$,

(c) $\|f\|_{X}+\left(\sum_{k=0}^{\infty}\left(q^{k \beta}\left\|U_{q^{k}} f-f\right\|_{X}\right)^{s}\right)^{1 / s}<\infty$.

Proof of Theorem 5. We show that the foregoing theorem can be applied to the following spaces and linear transformations. Let $X=L_{r}(\mathbf{K}), 1<r<2, Y=$ $\mathscr{D}\left(D_{r}^{[\alpha]}\right)$ for some $\alpha>0$ and, for $k \in N$ and $f \in X$, let $U_{k} f=\Delta_{n} f$ if $q^{n-1}<k<$ $q^{n}$. Clearly, $\left\{U_{k}\right\}_{1}^{\infty}$ is a sequence of bounded commutative linear transformations of $L_{r}(\mathbf{K})$, and we have (i) $\lim _{k \rightarrow \infty} U_{k} f=f$ for $f \in L_{r}(\mathbf{K})$. (ii) It follows from Lemma 1 and Theorem 3 that for each $f \in \mathscr{D}\left(D_{r}^{[\alpha]}\right)$ we have $f-\Delta_{n} * f=V_{-n, \alpha} * D_{r}^{[\alpha]} f$. Therefore,

$$
\left\|f-\Delta_{n} * f\right\|_{r} \leqslant\left\|V_{-n, \alpha}\right\|_{1}\left\|D_{r}^{[\alpha]} f\right\|_{r}<C q^{-n \alpha}\|f\|_{D(r, \alpha)} .
$$

(iii) According to Theorem 1 (c) for each $f \in L_{r}(\mathbf{K})$ and $n \in \mathbf{Z}$ we have $\Delta_{n} * f \in$ $\mathscr{D}\left(D_{r}^{[\alpha]}\right)$. Moreover, Theorem 1(b) immediately implies that

$$
\left\|D_{r}^{[\alpha]}\left(\Delta_{n} * f\right)\right\|_{r}=\left\|D_{r}^{[\alpha]}\left(\Delta_{n}\right) * f\right\|_{r}<C q^{n \alpha}\|f\|_{r}
$$


Thus

$$
\left\|\Delta_{n} * f\right\|_{D(r, \alpha)} \leqslant C q^{n \alpha}\|f\|_{r} .
$$

Consequently, the three conditions of the Butzer-Scherer theorem hold and we may conclude the validity of Theorem 5 .

5. Bessel potentials and Lipschitz spaces on $K$. In this section we first study the relationship between functions belonging to $\mathscr{D}\left(D_{r}^{[\alpha]}\right)$ and their Bessel potentials. Once this has been done we prove an additional result relating the spaces $\mathscr{D}\left(D_{r}^{[\alpha]}\right)$ and the Lipschitz spaces introduced in \$4. We repeat two definitions from [9] or [11].

Definition 3. For $f \in L_{1, \text { loc }}(\mathbf{K})$ its Littlewood-Paley function of order $p, 1<p$ $<\infty$, is defined by

$$
\mathcal{G}_{p} f(x)=\left(\sum_{l=-\infty}^{\infty}\left|\left(\Delta_{l}-\Delta_{l+1}\right) * f(x)\right|^{p}\right)^{1 / p} .
$$

Definition 4. For $f \in L_{r}(K), 1<r<\infty$, and $\alpha>0$ the function $J_{a} f$ is defined by $J_{\alpha} f=G_{\alpha} * f$. Furthermore, $L(r, \alpha)=\left\{f \in L_{r}(\mathbf{K}) ; f=J_{\alpha} g\right.$ for some $g \in$ $\left.L_{r}(\mathbf{K})\right\}$.

$J_{\alpha} f$ is called the Bessel potential of order $\alpha$ of $f$. If we define $\|f\|_{r, \alpha}=\|g\|_{r}$ if $f=J_{\alpha} g$, then $L(r, \alpha)$ is a Banach space with respect to $\|\cdot\|_{r, \alpha}$.

Our first result extends, at least for $1 \leqslant r \leqslant 2$, a theorem of Taibleson who proved that, for given $\alpha>0$ and $1 \leqslant r<\infty$, every $f \in \mathcal{S}$ belongs to $L(r, \alpha)$ with $\|f\|_{D(r, \alpha)} \simeq\|f\|_{r, \alpha}$, see [9, Corollary to Theorem 5].

TheOREM 6. Let $\alpha>0$ and $1 \leqslant r \leqslant 2$. Then $\mathscr{D}\left(D_{r}^{[\alpha]}\right)=L(r, \alpha)$ and the norms on these spaces are equivalent.

Proof. Take $f \in L(r, \alpha)$ and let $f=G_{\alpha} * h$ with $h \in L_{r}(\mathbf{K})$. For a.e. $y \in \mathbf{K}$ we have

$$
\hat{f}(y)= \begin{cases}\hat{h}(y) & \text { if } y \in P_{0}, \\ \|y\|^{-\alpha} \hat{h}(y) & \text { if } y \notin P_{0} .\end{cases}
$$

Thus, according to (3), we have $\|y\|^{\alpha} \hat{f}(y)=\left(\mu_{\alpha}\right)^{\wedge}(y) \hat{h}(y)$ a.e. on K. Consequently, there exists a $g \in L_{r}(\mathbf{K})$ with $\hat{g}(y)=\|y\|^{\alpha} \hat{f}(y)$ a.e. on $\mathbf{K}$, that is, $f \in W\left(L_{r},\|y\|^{\alpha}\right)$ $=\mathscr{D}\left(D_{r}^{[\alpha]}\right)$. Furthermore, a comparison of the Fourier transforms shows that

$$
h=g+\left(\Delta_{0}-D_{1}^{[\alpha]} \Delta_{0}\right) * f
$$

Therefore,

$$
\begin{aligned}
\|f\|_{r, \alpha} & =\|h\|_{r} \leqslant\|g\|_{r}+\left(\left\|\Delta_{0}\right\|_{1}+\left\|D_{1}^{[\alpha]} \Delta_{0}\right\|_{1}\right)\|f\|_{r} \\
& \leqslant C\left(\|g\|_{r}+\|f\|_{r}\right)=C\|f\|_{D(\alpha, r)} .
\end{aligned}
$$

Conversely, take $f \in \mathscr{D}\left(D_{r}^{[\alpha]}\right)$ and let $g=D_{r}^{[\alpha]} f$. Consider the function $h=g+$ $\left(\Delta_{0}-D_{1}^{[\alpha]} \Delta_{0}\right) * f$. Clearly, $h \in L_{r}(\mathbf{K})$ and we have

$$
\hat{h}(y)= \begin{cases}\hat{f}(y) & \text { if } y \in P_{0}, \\ \|y\|^{\alpha} \hat{f}(y) & \text { if } y \notin P_{0} .\end{cases}
$$


Thus $f=G_{\alpha} * h$ and, hence, $f \in L(r, \alpha)$. Also,

$$
\|f\|_{r} \leqslant\left\|G_{\alpha}\right\|_{1}\|h\|_{r}=\|h\|_{r}
$$

and

$$
\|g\|_{r} \leqslant\|h\|_{r}+\left(\left\|\Delta_{0}\right\|_{1}+\left\|D_{I}^{[\alpha]} \Delta_{0}\right\|_{1}\right)\|f\|_{r}<C\|h\|_{r} .
$$

Consequently,

$$
\|f\|_{D(r, \alpha)}=\|f\|_{r}+\|g\|_{r}<C\|h\|_{r}=C\|f\|_{r, \alpha} .
$$

This completes the proof of Theorem 6.

The next theorem is the analogue on $K$ of part of a well-known result of Taibleson in which he compares the spaces of Bessel potentials on $\mathbf{R}^{n}$ with the generalized Lipschitz spaces on $\mathbf{R}^{n}$, see [7, Chapter V] or [8].

THEOREM 7. For $\alpha>0$ and $1<r \leqslant 2$ we have

$$
\Lambda(\alpha, r, r) \subset L(r, \alpha)=\mathscr{D}\left(D_{r}^{[\alpha]}\right) \subset \Lambda(\alpha, r, 2) ;
$$

the inclusion mappings are continuous.

Proof. We first establish the second inclusion. Take any $f \in \mathscr{D}\left(D_{r}^{[\alpha]}\right)$ and let $g=D_{r}^{[\alpha]} f$. For each $k \in \mathbf{Z}$ we have

$$
q^{k \alpha}\left(\Delta_{k}-\Delta_{k+1}\right) * f=q^{-\alpha}\left(\Delta_{k}-\Delta_{k+1}\right) * g .
$$

Thus for the $\Lambda(\alpha, r, 2)$-norm of $f$ we have

$$
\begin{aligned}
\|f\|_{\Lambda(\alpha, r, 2)} & =\|f\|_{r}+\left(\sum_{k=-\infty}^{\infty}\left\|q^{k \alpha}\left(\Delta_{k}-\Delta_{k+1}\right) * f\right\|_{r}^{2}\right)^{1 / 2} \\
& =\|f\|_{r}+C\left(\sum_{k=-\infty}^{\infty}\left\|\left(\Delta_{k}-\Delta_{k+1}\right) * g\right\|_{r}^{2}\right)^{1 / 2} \\
& \leqslant\|f\|_{r}+C\left\|\left(\sum_{k=-\infty}^{\infty}\left|\left(\Delta_{k}-\Delta_{k+1}\right) * g\right|^{2}\right)^{1 / 2}\right\|_{r} \\
& =\|f\|_{r}+C\left\|\mathcal{S}_{2}(g)\right\|_{r} \\
& \leqslant\|f\|_{r}+C\|g\|_{r} \leqslant C\|f\|_{D(r, \alpha)} .
\end{aligned}
$$

The penultimate inequality is proved in Lemma 1.3 of [11, p. 197]. Thus, $f \in$ $\Lambda(\alpha, r, 2)$ and the identity mapping from $\mathscr{D}\left(D_{r}^{[\alpha]}\right)$ into $\Lambda(\alpha, r, 2)$ is continuous.

Now we consider the first inclusion of the theorem. Take $f \in \Lambda(\alpha, r, r)$. For $k \in \mathbf{Z}$ define $f_{k}$ by $f_{k}=\Delta_{k} * f$. Then $\lim _{k \rightarrow \infty} f_{k}=f$ in $L_{r}(\mathbf{K})$, and, according to Theorem 1(c), $f_{k} \in \mathscr{D}\left(D_{r}^{[\alpha]}\right)$ with

$$
D_{r}^{[\alpha]} f_{k}=\sum_{l=-\infty}^{k} q^{l \alpha}\left(\Delta_{l}-\Delta_{l-1}\right) * f
$$

In the following we prove that $\lim _{k \rightarrow \infty} D_{r}^{[\alpha]} f_{k}$ exists in $L_{r}(\mathbf{K})$. Since the operator $D_{r}^{[\alpha]}$ is a closed operator, see Corollary 2 , we may then conclude that $f \in \mathscr{D}\left(D_{r}^{[\alpha]}\right)$. For $s, k \in \mathbf{Z}$ with $s<k$ define $f_{s, k}$ by

$$
f_{s, k}=D_{r}^{[\alpha]} f_{k}-D_{r}^{[\alpha]} f_{s}=\sum_{l=s+1}^{k} q^{l \alpha}\left(\Delta_{l}-\Delta_{l-1}\right) * f .
$$


We first determine an estimate for the Littlewood-Paley function of order $r$ of the function $f_{s, k}$. Because for all $m, n \in \mathbf{Z}$ we have

$$
\left(\Delta_{m}-\Delta_{m+1}\right) *\left(\Delta_{n}-\Delta_{n+1}\right)=\delta_{m, n}\left(\Delta_{m+1}-\Delta_{m}\right)
$$

we see immediately that

$$
\left(\Delta_{l}-\Delta_{l+1}\right) * f_{s, k}= \begin{cases}0 & \text { if } l<s \text { or } l>k \\ q^{(l+1) \alpha}\left(\Delta_{l}-\Delta_{l+1}\right) * f & \text { if } s<l<k\end{cases}
$$

Consequently,

$$
\begin{aligned}
\mathcal{G}_{r}\left(f_{s, k}\right) & =\left(\sum_{l=-\infty}^{\infty}\left|\left(\Delta_{l}-\Delta_{l+1}\right) * f_{s, k}\right|^{r}\right)^{1 / r} \\
& =\left(\sum_{l=s}^{k-1}\left|q^{(l+1) \alpha}\left(\Delta_{l}-\Delta_{l+1}\right) * f\right|^{r}\right)^{1 / r} .
\end{aligned}
$$

Therefore,

$$
\begin{aligned}
\left\|\mathcal{G}_{r}\left(f_{s, k}\right)\right\|_{r}^{r} & =\int_{K} \sum_{l=s}^{k-1}\left|q^{(l+1) \alpha}\left(\Delta_{l}-\Delta_{l+1}\right) * f(x)\right|^{r} d x \\
& =\sum_{l=s}^{k-1}\left\|q^{(l+1) \alpha}\left(\Delta_{l}-\Delta_{l+1}\right) * f\right\|_{r}^{r}
\end{aligned}
$$

and we see that

$$
\left\|\mathcal{G}_{r}\left(f_{s, k}\right)\right\|_{r} \leqslant C\left(\|f\|_{\Lambda(\alpha, r, r)}-\|f\|_{r}\right) \text {. }
$$

Next, to obtain an estimate for $\left\|f_{s, k}\right\|_{r}$ we apply the converse of Hölder's inequality. Thus take $\varphi \in L_{r}(\mathbf{K})$ such that $\|\varphi\|_{r^{\prime}}=1$. Then, according to Lemma 20 in [10], we have

$$
\begin{aligned}
\left|\int_{K} f_{s, k}(x) \varphi(x) d x\right|= & \left|\int_{K} \sum_{l=-\infty}^{\infty}\left(\Delta_{l}-\Delta_{l+1}\right) * f_{s, k}(x)\left(\Delta_{l}-\Delta_{l+1}\right) * \varphi(x) d x\right| \\
< & \int_{K}\left(\sum_{l=-\infty}^{\infty}\left|\left(\Delta_{l}-\Delta_{l+1}\right) * f_{s, k}(x)\right|^{r}\right)^{1 / r} \\
& \cdot\left(\sum_{l=-\infty}^{\infty}\left|\left(\Delta_{l}-\Delta_{l+1}\right) * \varphi(x)\right|^{r^{\prime}}\right)^{1 / r^{\prime}} d x \\
= & \int_{K} \mathcal{G}_{r}\left(f_{s, k}\right)(x) \mathcal{G}_{r}(\varphi)(x) d x \\
< & \left\|\mathcal{G}_{r}\left(f_{s, k}\right)\right\|_{r}\left\|\mathcal{G}_{r^{\prime}}(\varphi)\right\|_{r^{\prime}}<C\left\|\mathcal{G}_{r}\left(f_{s, k}\right)\right\|_{r^{\circ}}
\end{aligned}
$$

The last inequality follows from Theorem 19 in [10] and the assumption that $\|\varphi\|_{r^{\prime}}=1$. Thus we have $\left\|f_{s, k}\right\|_{r}<C\left\|\mathcal{G}_{r}\left(f_{s, k}\right)\right\|_{r}$. Since $f \in \Lambda(\alpha, r, r)$, for each $\varepsilon>0$ there is an $M \in \mathbf{N}$ so that for all $s, k \in \mathbf{N}$ with $M<s<k$ we have

$$
\sum_{l=s}^{k}\left\|q^{l \alpha}\left(\Delta_{l}-\Delta_{l+1}\right) * f\right\|_{r}^{r}<\varepsilon
$$


Thus, it follows from (4) that $\left\|f_{s, k}\right\|_{r} \rightarrow 0$ as $s, k \rightarrow \infty$, and, hence, $\left\{D_{r}^{[\alpha]} f_{k}\right\}_{k=1}^{\infty}$ is a Cauchy sequence in $L_{r}(K)$. Consequently, $g=D_{r}^{[\alpha]} f$ exists, that is, $f \in \mathscr{D}\left(D_{r}^{[\alpha]}\right)$. Finally we prove the continuity of the identity mapping from $\Lambda(\alpha, r, r)$ into $\mathscr{D}\left(D_{r}^{[\alpha]}\right)$. We already proved that

$$
\left\|f_{s, k}\right\|_{r} \leqslant C\left\|\mathcal{G}_{r}\left(f_{s, k}\right)\right\|_{r} \leqslant C\left(\|f\|_{\Lambda(\alpha, r, r)}-\|f\|_{r}\right)
$$

for all $k, s \in \mathbf{Z}$. Since $D_{r}^{[\alpha]} f_{k}=\lim _{s \rightarrow-\infty} f_{s, k}$ for all $k \in \mathbf{N}$ we see that

$$
\left\|D_{r}^{[\alpha]} f_{k}\right\|_{r} \leqslant C\left(\|f\|_{\Lambda(\alpha, r, r)}-\|f\|_{r}\right) \text {. }
$$

Consequently,

$$
\|g\|_{r}=\left\|D_{r}^{[\alpha]} f\right\|_{r}<C\left(\|f\|_{\Lambda(\alpha, r, r)}-\|f\|_{r}\right)
$$

so that

$$
\|f\|_{D(r, \alpha)}=\|f\|_{r}+\|g\|_{r} \leqslant C\|f\|_{\Lambda(\alpha, r, r)} .
$$

This completes the proof of Theorem 7.

Our last result deals with the sharpness of the inclusion relations in Theorem 7. Like in the case of $\mathbf{R}^{n}$, see [7] or [8], in the local field setting Theorem 7 is best possible in the following sense.

THEOREM 8. Given $\alpha>0$ and $1<r<2$ we have

(i) if $s<2$ then $\mathscr{D}\left(D_{r}^{[\alpha]}\right) \nsubseteq \Lambda(\alpha, r, s)$,

(ii) if $s>r$ then $\Lambda(\alpha, r, s) \notin \mathscr{D}\left(D_{r}^{[\alpha]}\right)$.

Proof (Outline). (i) For $l \in \mathbf{N}$ let $y(l)=\mathfrak{p}^{-l}$ and let $\chi_{y(l)}$ be the corresponding character on $\mathbf{K}$. For $\alpha>0$ and $s<2$ define the functions $f$ and $g$ by

$$
\begin{aligned}
& f(x)=\Delta_{0}(x) \sum_{l=1}^{\infty} q^{-l \alpha} l^{-1 / s} \chi_{y(l)}(x) \\
& g(x)=\Delta_{0}(x) \sum_{l=1}^{\infty} l^{-1 / s} \chi_{y(l)}(x)
\end{aligned}
$$

Clearly $f, g \in L_{r}(\mathbf{K})$ and a long and tedious computation, in which we use the fact that the series defining $f$ is a lacunary series on $P_{0}$, shows that $G_{\alpha} * g(x)=f(x)$ a.e. on $\mathbf{K}$, so that $f \in L(r, \alpha)=\mathscr{D}\left(D_{r}^{[\alpha]}\right)$. Next, in order to estimate $\|f\|_{\Lambda(\alpha, r, s)}$ we first observe that for $l \geqslant 1$ we have

$$
\left(\Delta_{l}-\Delta_{l+1}\right) * f=\Delta_{0} q^{-l \alpha} l^{-1 / s} \chi_{y(l)}
$$

Therefore,

$$
\begin{aligned}
\|f\|_{\Lambda(\alpha, r, s)}^{s} & \geqslant \sum_{l=1}^{\infty}\left\|q^{l \alpha}\left(\Delta_{l}-\Delta_{l+1}\right) * f\right\|_{r}^{s} \\
& =\sum_{l=1}^{\infty}\left\|l^{-1 / s} \chi_{y(l)} \Delta_{0}\right\|_{r}^{s}=\sum_{l=1}^{\infty}\left(l^{-1 / s}\right)^{s}=\infty,
\end{aligned}
$$

that is, $f \notin \Lambda(\alpha, r, s)$. 
(ii) Consider the functions $f, g$ defined by

$$
\begin{aligned}
& f(x)=\sum_{l=1}^{\infty} q^{-l\left(\alpha+1 / r^{\prime}\right)} l^{-1 / r}\left(\Delta_{l}-\Delta_{l-1}\right)(x), \\
& g(x)=\sum_{l=1}^{\infty} q^{-l / r^{l} l^{-1 / r}}\left(\Delta_{l}-\Delta_{l-1}\right)(x) .
\end{aligned}
$$

Then $f \in L_{1}(\mathbf{K}) \cap L_{r}(\mathbf{K})$ and $g \in L_{1}(\mathbf{K})$ and a straightforward computation shows that $\hat{g}(y)=\|y\|^{\alpha} \hat{f}(y)$ a.e. on $\mathbf{K}$. Thus, if $f \in \mathscr{D}\left(D_{r}^{[\alpha]}\right)$ then we necessarily have $g=D_{r}^{[\alpha]} f$. However, another tedious computation shows that $\|g\|_{r}=\infty$ for $r>1$, so that $g \notin \mathscr{D}\left(D_{r}^{[\alpha]}\right)$. On the other hand, for each $k \in \mathbf{Z}$ we have

$$
\left(\Delta_{k+1}-\Delta_{k}\right) * f= \begin{cases}0 & \text { if } k<0, \\ q^{-(k+1)\left(\alpha+1 / r^{\prime}\right)}(k+1)^{-1 / r}\left(\Delta_{k+1}-\Delta_{k}\right) & \text { if } k>0,\end{cases}
$$

and $\left\|\Delta_{k}\right\|_{r}=q^{k / r^{\prime}}$. Thus we see that

$$
\begin{aligned}
\|f\|_{\Lambda(\alpha, r, s)} & \leqslant\|f\|_{r}+\left\{\sum_{k=0}^{\infty}\left(q^{k \alpha} q^{-(k+1)\left(\alpha+1 / r^{\prime}\right)}(k+1)^{-1 / r} 2 q^{k / r}\right)^{s}\right\}^{1 / s} \\
& \leqslant\|f\|_{r}+C\left(\sum_{k=0}^{\infty}(k+1)^{-s / r}\right)^{1 / s}<\infty,
\end{aligned}
$$

because $s>r$. Thus $f \in \Lambda(\alpha, r, s)$, which completes the proof of Theorem 8 .

\section{REFERENCES}

1. P. L. Butzer and H. Berens, Semi-groups of operators and approximations, Springer-Verlag, New York, 1967.

2. P. L. Butzer and K. Scherer, On the fundamental approximation theorems of D. Jackson, S. N. Bernstein and theorems of M. Zamansky and S. B. Stečkin, Aequationes Math. 3 (1969), 170-185.

3. P. L. Butzer and H. J. Wagner, Walsh-Fourier series and the concept of a derivative, Applicable Anal. 3 (1973), 29-46.

4. C. W. Onneweer, Differentation on a p-adic or p-series field, in Linear Spaces and Approximation, edited by P. L. Butzer and B. Sz.-Nagy, Birkhauser Verlag, Basel, 1978, pp. 187-198.

5. On the definition of dyadic differentiation, Applicable Anal. (to appear).

6. J. Pál, On the concept of a derivative among functions defined on the dyadic field, SIAM J. Math. Anal. 8 (1977), 375-391.

7. E. M. Stein, Singular integrals and differentiability properties of functions, Princeton Univ. Press, Princeton, N. J., 1970.

8. M. H. Taibleson, On the theory of Lipschitz spaces of distributions on Euclidean n-space, I. Principal properties, J. Math. Mech. 13 (1964), 407-480.

9. Harmonic analysis on n-dimensional vector spaces over local fields, I. Basic results on fractional integration, Math. Ann. 176 (1968), 191-207.

10. Harmonic analysis on n-dimensional vector spaces over local fields, II. Generalized Gauss kernels and the Littlewood-Paley function, Math. Ann. 186 (1970), 1-19.

11. _ Fourier analysis on local fields, Mathematical Notes, Princeton Univ. Press, Princeton, N. J., 1975.

Department of Mathematics, Universtty of New MeXico, Albuquerque, New MeXico 87131 